\title{
PI3K/AKT signaling pathway activation in a rat model of migraine
}

\author{
YUN-YONG LIU ${ }^{1}$, ZI-YAO JIAO ${ }^{2}$, WEI LI $^{1}$ and QIAN TIAN ${ }^{1}$ \\ Departments of ${ }^{1}$ Neurology and ${ }^{2}$ Anesthesiology, Linyi People's Hospital, Linyi, Shandong 276003, P.R. China
}

Received April 26, 2016; Accepted May 3, 2017

DOI: $10.3892 / \mathrm{mmr} .2017 .7191$

\begin{abstract}
The present study aimed to investigate phosphatidylinositol 4,5-bisphosphate 3-kinase (PI3K)/protein kinase B (AKT) signaling pathway activation in a rat model of migraine. A total of 60 male Sprague-Dawley rats were randomly divided into three groups: Blank control; suspension control; and migraine model. The model group was subcutaneously injected with a glyceryl trinitrate suspension, using an optimized Tassorelli method to establish a rat model of migraine. The activation status of the PI3K/AKT signaling pathway was assessed via measurement of the phosphorylated (p)-AKT level. The level of serum 5-hydroxytryptamine was detected using an ELISA. The mRNA and protein expression levels of PI3K and AKT, and protein levels of p-AKT were detected by reverse transcription quantitative polymerase chain reaction and western blot analysis. Expression of the PI3K gene was significantly increased $(\mathrm{P}<0.01) 6-24 \mathrm{~h}$ following the glyceryl trinitrate injection. There was no significant difference in the expression of AKT between any of the groups at any time. Expression of p-AKT (S473) was significantly increased in the migraine model group $(\mathrm{P}<0.01)$ compared with the controls groups. Immunohistochemical analysis indicated that phosphatase and tensin homolog (PTEN) continuously decreased in the migraine model group during 1-12 $\mathrm{h}$, however this was only significant in the $12 \mathrm{~h}$ group. Levels of PTEN had increased again by $24 \mathrm{~h}$. Glycogen synthase kinase (GSK)-3 $\beta$ expression exhibited a similar expression pattern to PTEN. The results indicated that the PI3K/AKT signal pathway may be activated in the brain tissue of the rat migraine models. The inhibition of PTEN, which is an upstream modulator of the PI3K/AKT signaling pathway, may enhance the activation of phosphatidylinositol-3,4,5-triphosphate, thus inhibiting the expression of GSK-3 $\beta$.
\end{abstract}

Correspondence to: Ms. Qian Tian, Department of Neurology, Linyi People's Hospital, 27 Jie-Fang East Road, Lanshan, Linyi, Shandong 276003, P.R. China

E-mail: tianqian2016@sina.com

Key words: PI3K/AKT, migraine, Tassorelli method, signaling pathway

\section{Introduction}

Migraines are a primary central nervous system dysfunction that are commonly observed in the clinic; the majority of cases present with moderate and severe paroxysmal headache, accompanied by nausea and vomiting $(1,2)$. Notably, the headaches are aggravated by sound and light stimulation. Migraines are a common chronic neurological disorder, which are more frequent in women, children and adolescents. Susceptibility to migraines is considered to be a multifactorial and multigene disease, composed of environmental and genetic factors $(3,4)$. The molecular mechanism underlying migraines is currently clear. A previous study suggested that the phosphatidylinositol 4,5-bisphosphate 3-kinase (PI3K)/protein kinase B (AKT) signaling pathway is involved in a variety of physiological and pathological processes (5), however, there are limited studies investigating the activation of the PI3K/AKT signaling pathway in migraines.

The PI3K/AKT signaling pathway is an important survival signal transduction pathway in cells. It is widely involved in cell functions, including regulation of glucose transport and cell proliferation, differentiation and apoptosis. Following activation, PI3K generates the second messenger phosphatidylinositol-3,4,5-triphosphate (PIP3). AKT is a serine/threonine kinase, which can be activated by PIP3 following upstream activation of an associated tyrosine kinase receptor. Phosphorylated (p)-AKT further activates or inhibits downstream signaling molecules including $\mathrm{Bcl} 2$-associated agonist of cell death, caspase- 9 , nuclear factor- $\kappa B$, glycogen synthase kinase (GSK)-3 and forkhead box O1 by phosphorylation, and subsequently regulates proliferation, differentiation, apoptosis, migration and other biological processes.

The establishment of an animal model of migraine is an important tool to study the pathogenesis of migraines and the establishment of animal migraine models has recently been reported (6,7). The nitroglycerin-induced migraine model, which exhibits a similar time phase to human migraine and has a high success rate and operability, has been widely used in clinical research into migraines (8). The present study optimized the establishment of a nitroglycerin-induced migraine model. The model was evaluated by the detection of serum 5-hydroxytryptamine (5-HT) by ELISA. Activation of the PI3K/AKT signaling pathway was assessed via analysis of mRNA and protein expression levels of these genes. Alterations in the levels of downstream signal molecules were 
assessed in the process of migraine and provided experimental evidence for the clinical study of these proteins in the context of migraines.

\section{Materials and methods}

Materials. Sprague-Dawley male rats $(200 \pm 10 \mathrm{~g})$ were purchased from the Shanghai SLAC Laboratory Animal Co., Ltd. (Shanghai, China). Nitroglycerin was purchased from Beijing Yimin Pharmaceutical Co., Ltd. (Beijing, China). The 5-HT ELISA kit (cat. no. CEA808Ge) was purchased from Cloud-Clone Corp Company (Houston, TX, USA). TRIzol was purchased from Thermo Fisher Scientific, Inc. (Waltham, MA, USA). The PrimeScript RT reagent kit and SYBR Fast qPCR Mix were purchased from Takara Biotechnology Co., Ltd. (Dalian, China). The radioimmunoprecipitation assay (RIPA) lysis buffer and Bradford protein concentration determination kit were purchased from Beyotime Institute of Biotechnology (Haimen, China). Anti-AKT (cat. no. sc-8312), anti-p-AKT-Ser-473 (cat. no. sc-293125), anti-phosphatase and tensin homolog (PTEN; cat. no. sc-122834) and anti-GSK-3 $\beta$ (cat. no. sc-7291) monoclonal antibodies were purchased from Santa Cruz Biotechnology, Inc. (Dallas, TX, USA). The anti-GAPDH monoclonal antibody (cat. no. TA505454) and the horseradish peroxidase (HRP)-labeled secondary antibodies (cat. no. ZB-2305 and ZB-2306) were purchased from Beijing Zhongshan Jinqiao Biotechnology Co., Ltd. (Beijing, China). The enhanced chemiluminescence (ECL) detection kit was purchased from EMD Millipore (Billerica, MA, USA). The rabbit anti-mouse immunohistochemistry kit (cat. no. 2500023) was purchased from Abbiotec LLC (San Diego, CA, USA).

Creation of migraine model. Healthy adult male SD rats were maintained in standard animal cages (5 rats/cage). Rats were free to eat and drink water during the experiment. The temperature and relative humidity were maintained at $18-25^{\circ} \mathrm{C}$ and $40-70 \%$, respectively.

A total of 60 SD rats were randomly divided into the following three groups (n=20/group): Blank control group (BCG); suspension control group (SCG); and migraine model group (MMG). Nitroglycerin solution and vehicle were prepared for use in $50 \mathrm{ml}$ nitroglycerin syringes. A nitroglycerin suspension (nitrogen drying at room temperature and re-dissolution in $5 \mathrm{ml}$ ethanol; the volume was subsequently made up to $50 \mathrm{ml}$ with $1 \%$ Tween- 80 solution) was prepared by vortex mixing. The control suspension contained $5 \mathrm{ml}$ anhydrous ethanol made up to $50 \mathrm{ml}$ with $1 \%$ Tween- 80 solution. Subcutaneous injections of the nitroglycerin suspension $(9 \mathrm{mg} / \mathrm{kg})$ and the control suspensions were administered into the back of the rats' neck as a single dose. The blank control group did not receive any treatment. Serum samples for ELISA analysis were collected from the tail vein 1, 6, 12 and $24 \mathrm{~h}$ following the injection. The brain stem tissues were isolated and snap-frozen in liquid nitrogen. The present study was approved by the ethics committee of Linyi People's Hospital (Linyi, China).

Establishment of the rat migraine model. The model was established by injecting rats with a glyceryl trinitrate suspension (9).
The behaviors of the rats in first $2 \mathrm{~h}$ were recorded by video and counted by a researcher. Time bins $(20 \mathrm{~min})$ were selected according to a previous reference (9). There were 20 rats in each group. Behavioral changes in the rats were then observed, including periods of head and ear scratching, shaking the body and climbing the cage. The mean frequency of events was calculated across each time bin.

5-HT detection by ELISA. Blood samples were incubated at room temperature for $2 \mathrm{~h}$, and the serum was subsequently separated by centrifugation at $3,000 \mathrm{x} \mathrm{g}$ for $10 \mathrm{~min}$ at $4^{\circ} \mathrm{C}$. Measurement of 5-HT by ELISA was performed according to the manufacturer's protocol. The standards and diluted serum samples were added to the wells of the provided plate and incubated at $37^{\circ} \mathrm{C}$ for $2 \mathrm{~h}$. Test solution $(100 \mu \mathrm{l})$ was added to each well, a film cover was placed over the plate and the plate was incubated at $37^{\circ} \mathrm{C}$ for $2 \mathrm{~h}$. The plate was washed three times by sterile PBS, and $100 \mu \mathrm{l}$ of the ELISA kit HRP-labeled rabbit anti-mouse secondary antibody was added to each well and the plate was incubated at $37^{\circ} \mathrm{C}$ for $30 \mathrm{~min}$. Following incubation, $90 \mu$ l tetramethylbenzadine (TMB) substrate was added to each well, the plate was covered with a light-blocking film and subsequently incubated for a further $15-25 \mathrm{~min}$ at $37^{\circ} \mathrm{C}$. To visualize the TMB color change, $50 \mu 12 \mathrm{M} \mathrm{H}_{2} \mathrm{SO}_{4}$ was added to each well and the optical density was immediately measured at $450 \mathrm{~nm}$ using an ELISA plate reader.

Western blot analysis. Sample tissues were incubated with RIPA lysis buffer on crushed ice for $30 \mathrm{~min}$ and subsequently lyzed using short duration sonication for $10 \mathrm{sec}$, repeated 3 times, with a probe type ultrasonicator. The mixture was centrifuged at $15,000 \mathrm{x}$ g for $20 \mathrm{~min}$ at $4^{\circ} \mathrm{C}$ and the supernatant was transferred into a fresh centrifuge tube. The protein concentration was determined using a Bradford protein concentration kit.

Proteins ( $40 \mathrm{mg}$ ) were separated by $12 \%$ SDS-PAGE, and the gels were subsequently incubated in the transfer buffer for $10 \mathrm{~min}$. The 'sandwich' was assembled and proteins were transferred to polyvinylidene difluoride (PVDF) membranes at $100 \mathrm{~V}$ for $45-60 \mathrm{~min}$. The PVDF membranes were subsequently rinsed with TBS for 10-15 min, blocked with blocking buffer (cat. no. P0023B; Beyotime Institute of Biotechnology) for $1 \mathrm{~h}$ at $4^{\circ} \mathrm{C}$ and incubated with diluted primary antibodies [1:10; containing $1 \%$ skimmed milk diluted with TBS-Tween (TBST)] at room temperature for $2 \mathrm{~h}$. The membranes were subsequently rinsed with TBST for 5-10 min three times, and incubated with rabbit anti-mouse secondary antibody $(1: 10,000)$, diluted in TBST containing $0.05 \%$ skimmed milk. The membranes were rinsed with TBST for 5-10 min three times. ECL substrate was added and the membranes were exposed in a darkroom.

Reverse transcription quantitative polymerase chain reaction (RT-qPCR) analysis. Tissue samples were ground in liquid nitrogen. Total RNA was extracted using TRIzol (with gDNA Eraser), and the concentration and purity were detected using a Nanodrop 2000 (Thermo Fisher Scientific, Inc., Wilmington, DE, USA). Total RNA was reversed transcribed to cDNA according to the manufacturer's protocol. qPCR was performed to detect mRNA expression levels. Primers were designed and 
Table I. Primers for reverse transcription quantitative polymerase chain reaction.

\begin{tabular}{lllrr}
\hline Gene & \multicolumn{1}{c}{$\begin{array}{c}\text { GenBank } \\
\text { accession no. }\end{array}$} & \multicolumn{1}{c}{ Primer (5'-3') } & Length & $\mathrm{T}_{\mathrm{m}}$ \\
\hline PI3K & NM_001024955 & F: ACACCACGGTTTGGACTATGG & 21 & 62.0 \\
& & R: GGCTACAGTAGTGGGCTTGG & 20 & 62.0 \\
AKT & NM_028420 & F: ATGTCCGAGATCCTACCCTACG & 22 & 62.2 \\
& & R: AGCGAAGAAGGAGTTGGTGTC & 21 & 62.0 \\
GAPDH & NM_008084.3 & F: AGGTCGGTGTGAACGGATTTG & 21 & 61.0 \\
& & R: GGGGTCGTTGATGGCAACA & 19 & 61.0 \\
\hline
\end{tabular}

F, forward; R, reverse; PI3K, phosphatidylinositol-3-kinase; AKT, protein kinase B; $\mathrm{T}_{\mathrm{m}}$, melting temperature.

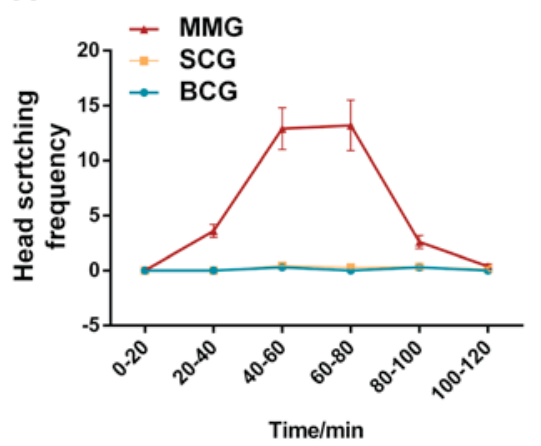

B

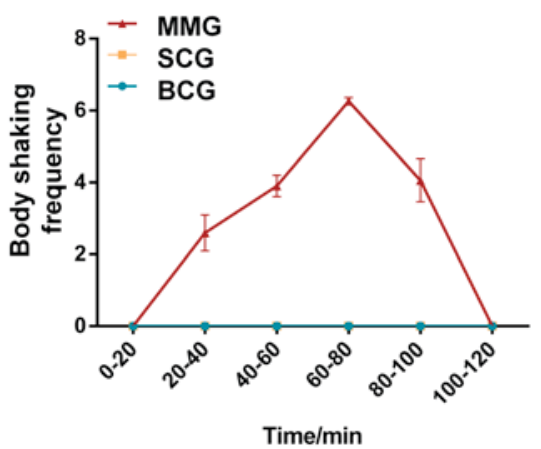

C

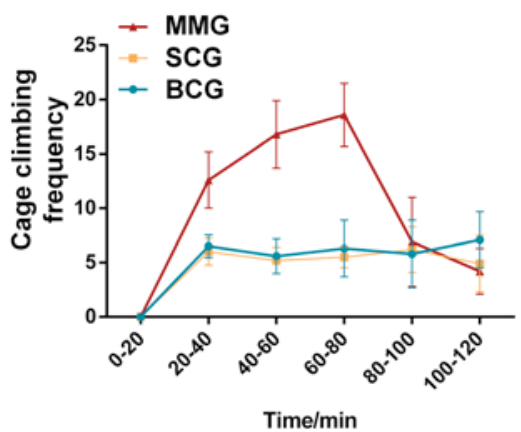

Figure 1. Behavioral measurements in MMG, SCG and BCG rats. (A) Head scratching, (B) body shaking and (C) cage climbing was monitored. Data are presented as the mean \pm standard deviation ( $n=20$ /group). MMG, migraine model group; SCG, suspension control group; BCG, blank control group.

synthesized by Shanghai SaiBaiSheng Gene Technology Co., Ltd. (Shanghai, China). The specific sequences are provided in Table I. The $\mathrm{Cq}$ value for each gene was measured, and the $\Delta \Delta \mathrm{Cq}$ values were determined from the average of three parallel experiments. The values were calculated as follows: $\Delta \mathrm{Cq}=\mathrm{Cq}_{\text {(Target Gene) }}-\mathrm{Cq}_{\text {(Reference gene) }} ; \Delta \Delta \mathrm{Cq}=\Delta \mathrm{Cq}_{\text {(sample) }}-\Delta \mathrm{Cq}_{\text {(control). }}$ The relative expression level of target genes (PI3K and AKT) was calculated using the $2^{-\Delta \Delta C q}$ method (10). The relative expression level of the control (GAPDH) was $2^{\circ}=1$. The $q P C R$ reactions contained: $10 \mu \mathrm{l} 2 \mathrm{X}$ SYBR Fast qPCR mix; $0.8 \mu \mathrm{l}$ PCR forward/reverse Primers (10 mM); $0.4 \mu 1$ 50X ROX reference dye II; $2 \mu \mathrm{l}$ cDNA template. The reaction parameters were as follows: Initial denaturation at $95^{\circ} \mathrm{C}$ for $30 \mathrm{sec}$, followed by 40 cycles of $95^{\circ} \mathrm{C}$ for $3 \mathrm{sec}$ and $60^{\circ} \mathrm{C}$ for $13 \mathrm{sec}$.

Immunohistochemistry. Brain stem tissue samples were fixed with $10 \%$ neutral buffered formalin for $24 \mathrm{~h}$ at room temperature, and then washed with tap water. The samples were dehydrated with an alcohol gradient, embedded using paraffin and cut into $4.5 \mathrm{~mm}$ slices. The sections were dewaxed using dimethylbenzene at room temperature, followed by graded alcohol, pure water and PBS. Immunohistochemical staining was performed to detect the levels of PTEN (1:400) and GSK-3 $\beta$ (1:200) for $20 \mathrm{~min}$ at $98^{\circ} \mathrm{C}$, using monoclonal antibodies and an immunohistochemistry kit, according to the manufacturer's instructions (Abbiotec LLC). Immunohistochemical data were analyzed using Image-Pro Plus software version 6.0 (Media Cybernetics, Inc., Rockville, MD, USA). The area of interest was selected and the IOD (integrated optic density) was measured. The IOD/area (density mean) of the selected area was calculated, and the density mean value was used to represent the semi-quantitative protein expression level.

Statistical analysis. All analyses were performed using SPSS version 13.0 (SPSS, Inc., Chicago, IL, USA). The mean and standard deviation were used to summarize continuous variables. One-way analysis of variance and Fisher's least significant difference post hoc test were used to assess the differences among groups. $\mathrm{P}<0.05$ was considered to indicate a statistically significant difference.

\section{Results}

Establishment of the rat migraine model. The model was established by injecting rats with a glyceryl trinitrate suspension ( $n=20$ rats/group). Behavioral changes in the rats were observed, including periods of head and ear scratching, shaking the body and climbing the cage. MMG rats scratched more frequently than SCG and BCG and rats. All MMG rats exhibited red ears, cage climbing and scratching 5 min following model establishment. The behavioral changes of the rats across $2 \mathrm{~h}$ following model induction are summarized in Fig. 1, which presents the average frequency of the event within each time duration bin. There were no significant differences between the BCG and the SCG rats. However, there were significant differences in the frequency of scratching, body shaking and 
Table II. Concentrations of serum 5-HT ELISA.

\begin{tabular}{lcccr}
\hline $\begin{array}{l}\text { Time point following } \\
\text { injection, h }\end{array}$ & $\begin{array}{c}\text { Blank control } \\
\text { group }\end{array}$ & $\begin{array}{c}\text { Suspension } \\
\text { control group }\end{array}$ & $\begin{array}{c}\text { Migraine } \\
\text { model group }\end{array}$ & P-value \\
\hline 1 & $215.10 \pm 10.24$ & $199.25 \pm 13.94$ & $136.21 \pm 15.11^{\mathrm{a}, \mathrm{b}}$ & $<0.01$ \\
6 & $206.13 \pm 11.38$ & $209.54 \pm 14.65$ & $127.09 \pm 14.05^{\mathrm{a}, \mathrm{b}}$ & $<0.05$ \\
12 & $209.21 \pm 13.24$ & $217.09 \pm 11.37$ & $158.11 \pm 12.58^{\mathrm{a}, \mathrm{b}}$ & $<0.05$ \\
24 & $219.37 \pm 13.08$ & $223.34 \pm 15.21$ & $186.52 \pm 19.66^{\mathrm{a}, \mathrm{b}}$ & $<0.01$ \\
\hline
\end{tabular}

${ }^{\mathrm{a}}$ vs. the blank control group; ${ }^{\mathrm{b}} \mathrm{vs}$. the suspension control group. 5-HT, serotonin.
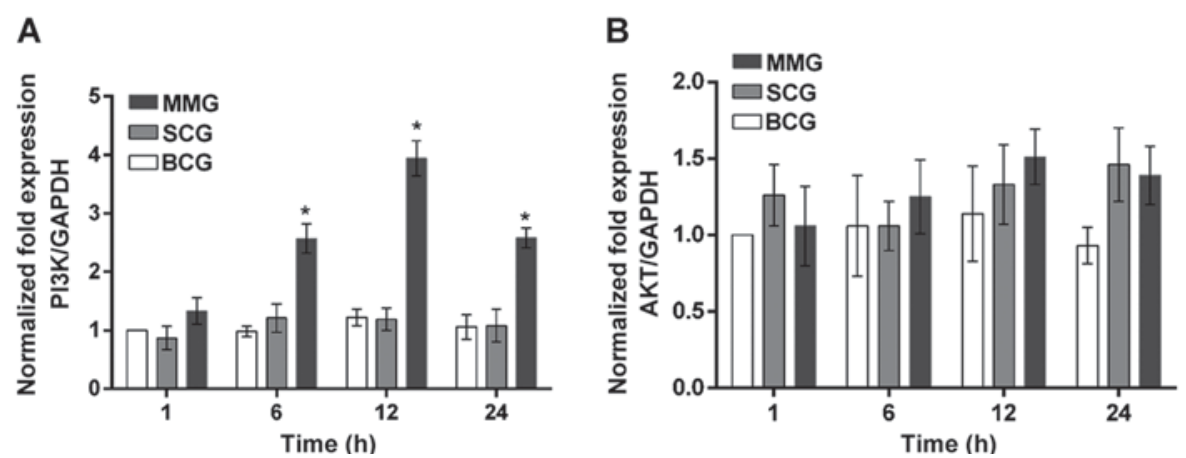

C

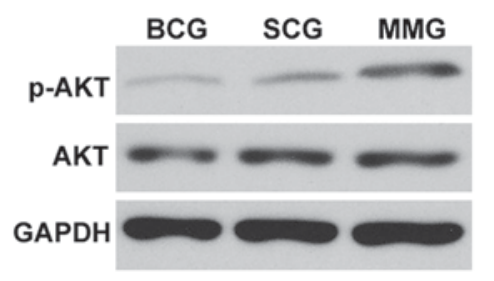

Figure 2. PI3K/AKT signal pathway activation in MMG, SCG and BCG rats. (A) PI3K and (B) AKT mRNA expression levels were measured using the reverse transcription quantitative polymerase chain reaction. (C) p-AKT and AKT protein expression levels were assessed by western blot analysis. Data are presented as the mean \pm standard deviation (n=20/group). "P<0.05 vs. BCG. MMG, migraine model group; SCG, suspension control group; BCG, blank control group; PI3K, phosphatidylinositol-3-kinase; p, phosphorylated; AKT, protein kinase B.

cage climbing between the MGG and the SCG rats $(\mathrm{P}<0.01)$, indicating that treatment with the glyceryl trinitrate suspension significantly altered the behavior of the rats.

Serum 5-HT is considered to be an endogenous index for evaluating migraine models (11). Blood samples were collected from the tail vein and the serum was separated, at 1, 6, 12 and $24 \mathrm{~h}$ following injection. The serum 5-HT levels were detected by ELISA (Table II). The measurements collected from BCG rats served as the baseline value. The results indicated that serum 5-HT decreased significantly in MMG rats, compared with the $\mathrm{BCG}$ rats $(\mathrm{P}<0.05)$.

PI3K/AKT signaling pathway activation in the rat migraine model. The level of p-AKT was measured to assess activation of the PI3K/AKT signaling pathway. Brain stem tissues were isolated at 1, 6, 12 and $24 \mathrm{~h}$ following injection of glyceryl trinitrate suspension. RT-qPCR was used to investigate the association of migraine occurrence with the expression levels of PI3K and AKT (Fig. 2A and B). There were no significant differences in the expression of PI3K and AKT mRNA across all three treatment groups within $1 \mathrm{~h}$ of the glyceryl trinitrate injection. However, the expression of PI3K was significantly increased in the MMG rats compared with the SCG and BCG rats $(\mathrm{P}<0.01), 6-24 \mathrm{~h}$ following the model induction. The level of AKT gene expression was not significantly different between any of the treatment groups at any time point. The expression of p-AKT was detected by western blot analysis at $12 \mathrm{~h}$ following the glyceryl trinitrate injection. The results (Fig. 2C) indicated that the expression of the activated form
p-AKT (S473) was higher in MMG rats compared with SCG and $\mathrm{BCG}$ rats.

Association between PI3K/AKT pathway and migraine of rats. To further investigate the association between PI3K/AKT signaling pathway activation and migraine occurrence in rats, the expression of PTEN and its downstream molecule, GSK-3 $\beta$, were evaluated by immunohistochemistry. All of the brain stem tissue samples acquired at 1, 6, 12 and $24 \mathrm{~h}$ following glyceryl trinitrate injection were subsequently formalin-fixed and embedded into paraffin blocks. The density mean of the selected area was used to semi-quantitatively assess the target protein expression level. The results indicated that the expression of PTEN continuously decreased in the MMG rats from 1-12 h, and increased again by $24 \mathrm{~h}$, however the difference between the groups was only significant at $12 \mathrm{~h}$ (Fig. 3). GSK-3 $\beta$ exhibited a similar alteration in expression to PTEN; there was a significant difference between the MMG rats and the SCG and BCG rats at 6,12 and $24 \mathrm{~h}$ post glyceryl trinitrate injection (Fig. 3).

\section{Discussion}

Migraine pathogenesis is complex, and the factors that influence its pathophysiology are diverse. There are several vasomotor related theories that explain the migraine mechanism, including the cortical spreading depression theory, the trigeminovascular system theory and the nitric oxide receptor theory (12-14). Activation of the trigeminovascular 

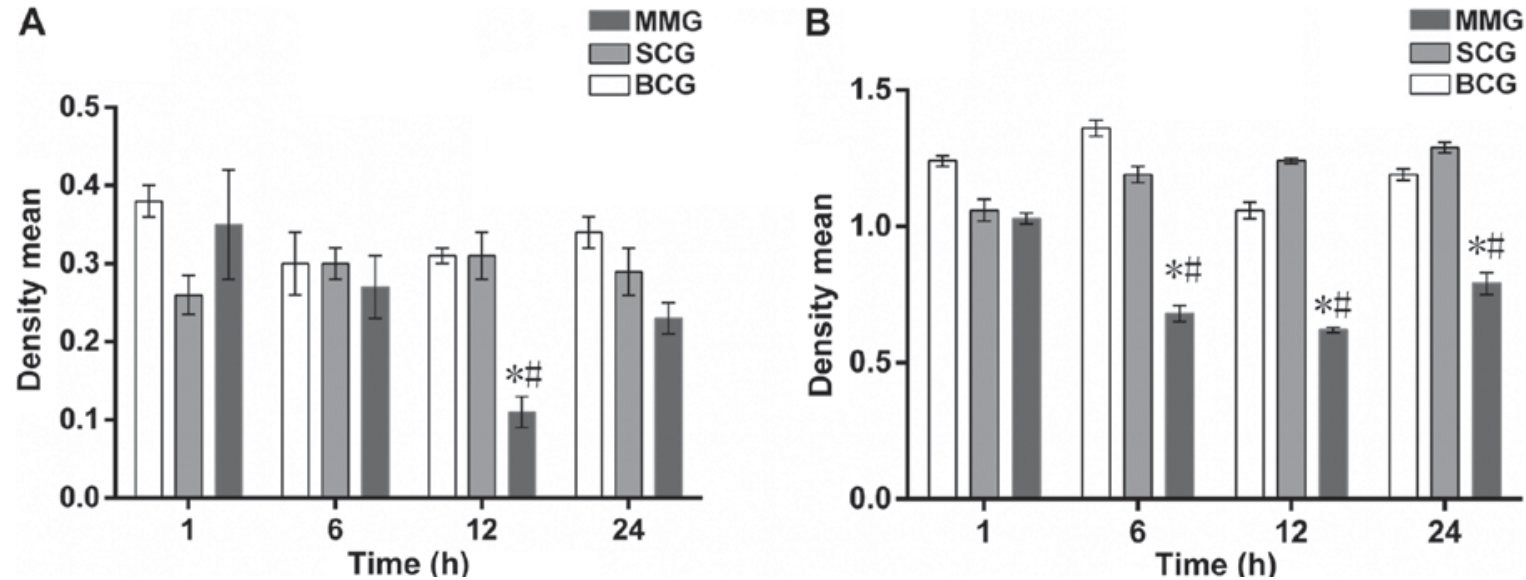

Figure 3. PTEN and GSK-3 $\beta$ expression in MMG, SCG and BCG rats. (A) PTEN and (B) GSK-3 $\beta$ expression levels were determined by immunohistochemistry. Data are presented as the mean \pm standard deviation ( $\mathrm{n}=20 /$ group). ${ }^{*} \mathrm{P}<0.05 \mathrm{vs}$. BCG; ${ }^{\text {"P }}<0.05$ vs. SCG. MMG, migraine model group; SCG, suspension control group; BCG, blank control group.

system leading to dual aseptic inflammation is a key link in migraine pathogenesis; abnormal activation of nerves associated with the vascular system may lead to migraine development (15). Numerous studies have focused on the molecular mechanism of the occurrence and development of migraines, and the results indicated that several signaling pathways are involved in the neural transmission process of migraines. Huang et al (16) revealed that extracellular signal-related kinase (ERK), which is an important marker of excited nociceptive neurons, serves an important role in pain stimulated nerve conduction. Other studies have also demonstrated similar results, whereby the abnormal expression of trigeminal nerve p-ERK served a significant role in nociceptive stimulation conduction (17). Recent studies have indicated that crosstalk between the AKT and ERK signaling pathways is crucial in the coordination of the nerve signal transmission and occurrence of migraines (18-20). The PI3K/AKT signaling pathway is one of the most important intracellular signal transduction pathways, affecting the activity of several downstream effector molecules. Levels of p-AKT reflect the activation status of the PI3K/AKT signaling pathway. The activation of AKT is achieved by the combination of the pleckstrin homology domain on AKT with PIP3. In addition, the T308 site on AKT is phosphorylated by phosphoinositide-dependent kinase 1, and this results in significant upregulation of AKT activity. However, complete activation of AKT requires phosphorylation of the S473 site (21). Therefore, the present study investigated the phosphorylation level of AKT S473.

The expression of PI3K mRNA and p-AKT were significantly higher in the MMG rats, compared with the control groups, at 6-24 h post injection. Although the total amount of AKT mRNA demonstrated no significant differences in the brain stem tissue of rats in each group, its activated form, p-AKT (S473), was expressed at significantly higher levels in the model group at $12 \mathrm{~h}$ post injection, indicating that the PI3K/AKT signaling pathway was abnormally activated in the MMG rats. Furthermore, PTEN was continuously downregulated in MMG rats from 1-12 h; however, this was only significant at $12 \mathrm{~h}$. PTEN levels appear to increase again by $24 \mathrm{~h}$. The expression trend of GSK-3 $\beta$ was similar to the trend observed for PTEN. PTEN inhibits the PI3K-mediated phosphorylation of phosphatidylinositol 4,5-bisphosphate; therefore, downregulation of PTEN in the model group may result in abnormal activation of AKT. Furthermore, activated AKT may negatively regulate the expression and activation of GSK-3 $\beta$. The specific molecular mechanism of this process and its role in the development of migraine require further biochemical investigation.

The nitroglycerin induced migraine rat model utilizes the decomposition of nitroglycerin into NO in the body, which induces a headache. The model is economical and simple, the set-up time is short and the pathological and biochemical changes are similar with the characteristics of a human migraine attack, therefore, it has been widely used in the construction of animal migraine models $(8,22)$. However, a previous study demonstrated that nitroglycerin may significantly dilate blood vessels and cause expansion of the subcutaneous capillaries over a short period of time. This stimulation may hinder the effective absorption of nitroglycerin, thereby exaggerating the differences observed in the model (9). The present study improved the nitroglycerin administration method by preparing a nitroglycerin mixed suspension, which did not cause rat mortality. The rats in the model group exhibited red ears, climbed their cages and scratched theirs heads and ears within 5 min of the model induction. Behaviors were observed for the first $2 \mathrm{~h}$ following nitroglycerine injection to confirm the success of migraine model.

To investigate the animal model, behavioral changes and ELISA analysis of serum 5-HT levels were recorded. Within $2 \mathrm{~h}$ of the nitroglycerine injection, the behavior of the BCG and SCG rats exhibited no significant differences, however, the MMG rats displayed significantly different behavior, compared with the control groups in the frequency of head and ear scratching, body shaking and cage climbing, indicating that the nitroglycerin was able to significantly alter the behavior of the MMG rats. 5-HT is an autacoid that is typically stored in the cell vesicles together with ATP; $90 \%$ of this chemical is synthesized and distributed by the enterochromaffin cells. Under the effects of stimulating factors, 5-HT is released from the granule cells and dispersed into the blood, and may be involved in the regulation of physiological functions including pain, sleep and body temperature (23). Furthermore, 5-HT 
content is associated with the occurrence of psychological disorders and migraine (24). When pathological and genetic factors result in abnormal dilatation of brain blood vessels, 5 -HT is released to 'buffer' the stress response. Therefore, it is an appropriate indicator for an observational index of the migraine model (25). The experimental results of the present study indicated that the serum 5-HT levels of the MMG rats rose significantly following induction of the model.

A significant limitation of the study involves the large time interval of measurements for the biochemical measurements; data was not acquired in the 1-6 h post injection time. Due to the long interval in biochemical measurements at an early point in the study, the present study may not provide strong support for the proposed migraine mechanism model in rat brain stem tissues. While the present study has demonstrated the role of the PI3K/AKT signaling pathway in a rat model of migraine, further research into the molecular mechanisms of the development of migraine are required in the future.

\section{References}

1. Chojnacka K, Zarzycka M, Hejmej A, Mruk DD, Gorowska E, Kotula-Balak M, Klimek M and Bilinska B: Hydroxyflutamide affects connexin 43 via the activation of PI3K/Akt-dependent pathway but has no effect on the crosstalk between PI3K/Akt and ERK1/2 pathways at the Raf-1 kinase level in primary rat Sertoli cells. Toxicol In Vitro 31: 146-157, 2016.

2. Petrulea MS, Plantinga TS, Smit JW, Georgescu CE and Netea-Maier RT: PI3K/Akt/mTOR: A promising therapeutic target for non-medullary thyroid carcinoma. Cancer Treat Rev 41: 707-713, 2015.

3. Jensen R and Stovner LJ: Epidemiology and comorbidity of headache. Lancet Neurol 7: 354-361, 2008.

4. Manandhar K, Risal A, Linde M and Steiner TJ: The burden of headache disorders in Nepal: Estimates from a population-based survey. J Headache Pain 17: 3, 2015.

5. Jin D, Yang JP, Hu JH, Wang LN and Zuo JL: MCP-1 stimulates spinal microglia via PI3K/Akt pathway in bone cancer pain. Brain Res 1599: 158-167, 2015.

6. Greco R, Bandiera T, Mangione AS, Demartini C, Siani F, Nappi G, Sandrini G, Guijarro A, Armirotti A, Piomelli D and Tassorelli C: Effects of peripheral FAAH blockade on NTG-induced hyperalgesia-evaluation of URB937 in an animal model of migraine. Cephalalgia 35: 1065-1076, 2015.

7. Hansen EK and Olesen J: Towards a pragmatic human migraine model for drug testing: 2. Isosorbide-5-mononitrate in healthy individuals. Cephalalgia 37: 11-19, 2016.

8. Tassorelli C, Greco R, Wang D, Sandrini M, Sandrini G and Nappi G: Nitroglycerin induces hyperalgesia in rats-a time-course study. Eur J Pharmacol 464: 159-162, 2003.
9. Jiang W, Wei HM, Zhao M, Guo S and Liu XY: Improvement of migraine model in rats and its evaluation indexes. Prog Modern Biomed 15: 1623-1627, 2015 (In Chinese).

10. Livak KJ and Schmittgen TD: Analysis of relative gene expression data using real-time quantitative PCR and the 2(-Delta Delta C(T)) Method. Methods 25: 402-408, 2001.

11. Arulmozhi DK, Veeranjaneyulu A and Bodhankar SL: Migraine: Current concepts and emerging therapies. Vascul Pharmacol 43: 176-187, 2005.

12. Mendes-da-SilvaRF,Lopes-de-Morais AA,Bandim-da-SilvaME, Cavalcanti Gde A, Rodrigues AR, Andrade-da-Costa BL and Guedes RC: Prooxidant versus antioxidant brain action of ascorbic acid in well-nourished and malnourished rats as a function of dose: A cortical spreading depression and malondialdehyde analysis. Neuropharmacology 86: 155-160, 2014.

13. Messlinger K, Lennerz JK, Eberhardt M and Fischer MJ: CGRP and NO in the trigeminal system: Mechanisms and role in headache generation. Headache 52: 1411-1427, 2012.

14. Walker CS and Hay DL: CGRP in the trigeminovascular system: A role for CGRP, adrenomedullin and amylin receptors? Br J Pharmacol 170: 1293-1307, 2013.

15. Dalkara T, Zervas NT and Moskowitz MA: From spreading depression to the trigeminovascular system. Neurol Sci 27 (Suppl 2): S86-S90, 2006.

16. Huang WJ, Wang BR, Yao LB, Huang CS, Wang X, Zhang P, Jiao XY, Duan XL, Chen BF and Ju G: Activity of p44/42 MAP kinase in the caudal subnucleus of trigeminal spinal nucleus is increased following perioral noxious stimulation in the mouse. Brain Res 861: 181-185, 2000.

17. Seino D, Tokunaga A, Tachibana T, Yoshiya S, Dai Y, Obata K, Yamanaka H, Kobayashi K and Noguchi K: The role of ERK signaling and the $\mathrm{P} 2 \mathrm{X}$ receptor on mechanical pain evoked by movement of inflamed knee joint. Pain 123: 193-203, 2006.

18. Guan XH, Fu QC, Shi D, Bu HL, Song ZP, Xiong BR, Shu B, Xiang HB, Xu B, Manyande A, et al: Activation of spinal chemokine receptor CXCR3 mediates bone cancer pain through an Akt-ERK crosstalk pathway in rats. Exp Neurol 263: 39-49, 2015.

19. Matsuzaki S and Darcha C: Co-operation between the AKT and ERK signaling pathways may support growth of deep endometriosis in a fibrotic microenvironment in vitro. Hum Reprod 30: 1606-1616, 2015.

20. Srivastava S, Sharma K, Kumar N and Roy P: Bradykinin regulates osteoblast differentiation by $\mathrm{Akt} / \mathrm{ERK} / \mathrm{NF \kappa B}$ signaling axis. J Cell Physiol 229: 2088-2105, 2014.

21. Abeyrathna P and Su Y: The critical role of Akt in cardiovascular function. Vascul Pharmacol 74: 38-48, 2015.

22. Ferrari LF, Levine JD and Green PG: Mechanisms mediating nitroglycerin-induced delayed-onset hyperalgesia in the rat. Neuroscience 317: 121-129, 2016.

23. de Herder WW: Biochemistry of neuroendocrine tumours. Best Pract Res Clin Endocrinol Metab 21: 33-41, 2007.

24. Lesurtel M, Graf R, Aleil B, Walther DJ, Tian Y, Jochum W, Gachet C, Bader M and Clavien PA: Platelet-derived serotonin mediates liver regeneration. Science 312: 104-107, 2006.

25. Jiang W, Wei HM, Zhao M, Guo S and Liu XY: Improvement of migraine model in rats and its evaluation indexes. Advances in Modern BIology 9: 1623-1627, 2015. 